\title{
Educational Software based on Augmented Reality: A Systematic Literature Review
}

\author{
Eduardo J. Sosa ${ }^{a}$, Raúl A. Aguilar, ${ }^{\mathrm{a},}$, José L. López ${ }^{\mathrm{b}}$, Omar S. Gómez ${ }^{\mathrm{c}}$ \\ ${ }^{a}$ Facultad de Matemáticas, Universidad Autónoma de Yucatán, Periférico Norte Tablaje 13615, A.P. 172, Mérida, C.P. 97110, México \\ ${ }^{b}$ Unidad Multidiciplinaria Tizimín, Universidad Autónoma de Yucatán, Calle 48 S/N, Tizimín, C.P. 97700, México \\ ${ }^{c}$ GrIISoft Research Group, Escuela Superior Politéctica de Chimborazo, Riobamba 060106, Chimborazo, Ecuador \\ Corresponding Author: *avera@correo.uady.mx
}

\begin{abstract}
Augmented Reality (AR) is a technology that has been seen to have potential in the educational sector for some years due to the tremendous positive impact that it could generate when used in teaching and learning processes. However, to achieve this, it is essential to consider educational and technological aspects to develop appropriate applications that provide the student and the teacher with innovative teaching methods. For this reason, this study presents a Systematic Review of Literature (SRL) under the protocol proposed by Kitchenham \& Stuart (2007) to collect relevant information that provides new research opportunities. This review focuses on augmented reality's technological aspect since the authors previously conducted an initial study considering the educational aspect. As part of this SRL, four sources of information were consulted: IEEE, ACM, Springer, and Elsevier, from which forty-two primary studies were analyzed that describe educational initiatives that incorporate Augmented Reality technology as part of their instructional design in the last decade. Finally, for this study's purposes, information is reported about the devices and mechanisms of interaction, tools and programming languages, recognition models, and the scarcity of methodologies used to develop educational applications with Augmented Reality is identified. In this way, an opportunity arises for research and innovation in the field of Software Engineering Methods and Models.
\end{abstract}

Keywords - Augmented reality; educational software; systematic review of literature.

Manuscript received 7 Nov. 2020; revised 16 Feb. 2021; accepted 3 Mar. 2021. Date of publication 31 Aug. 2021. IJASEIT is licensed under a Creative Commons Attribution-Share Alike 4.0 International License.

\section{INTRODUCTION}

Educational Informatics is a discipline that seeks the appropriate use of information technologies during the educational and learning process to obtain positive effects on students; said discipline studies the use, effect, and consequences of information technology and the educational process [1]. One of the research lines that has boomed in the last two decades - in educational informatics - is the use of Augmented Reality technology as a disruptive element to promote learning. Augmented Reality technology allows physical and digital information to be combined in real-time through different technological supports [2]. Thus, creating a new enriched reality with which the learner can interact.

In the field of education, interaction turns out to be a necessary mechanism for developing competencies in the learner. That is why it would be expected that Augmented Reality would potentiate instructional strategies that incorporate applications based on said technology. In order to gain a better insight on the application of augmented reality in education, this secondary research reports the results of a Systematic Literature Review on experiences of the application of augmented reality in educational initiatives, where authors are particularly interested in aspects related to the technological factor.

\section{A. Augmented Reality Technology}

Augmented Reality (AR) is an emerging technology that has gained popularity in recent years [3]. In Virtual Reality (VR) technology, the user experiences an immersion in the virtual environment generated by a software system and interacts with it through peripheral devices. AR extends the interaction capabilities of the individual by creating a connection between the real world in which it is found and digital objects generated with a software system, which are superimposed on or composed of real-world objects. AR is a technology that complements perception and interaction with the real world and allows the user to be in a real environment augmented with additional information generated by the 
computer [4]. According to Azuma [5], an AR system must have three elemental characteristics:

- Combine the real with the virtual world.

- Provide interaction in real-time.

- Keep a track in a three-dimensional plane.

Concerning the connection of the real world with objects or virtual environments, AR has used the four recognition methods described below:

- Pattern: this method uses a basic shape or marker incorporated into the object with which the subject is going to interact [6].

- Contour is a method by which an object or body, or part of it, is recognized and combined with some digital object.

- Surface: uses touch screens or a combination of projections on flat surfaces (e.g., walls, floors, etc.) with image recognition mechanisms that give, in realtime, the personal feeling of interaction with the projected objects [7].

- Location: This method identifies the location of the individual based on a virtual environment generated that links information from the real environment in which the individual is located; from the above, the real environment is fed with digital objects.

Interaction in virtual educational environments can be defined as an intrinsic characteristic of educational practice in the sense of social communication [8]. However, it is also an inherent property of any interactive virtual environment that includes physical, sensory, and mental activities and responses. In an AR application, the interaction necessarily requires an exchange of information between two entities through some interface, which is why this process can be analyzed from two perspectives: the interaction in which the learner is the issuer, and the AR system assumes the issuer role.

From the first perspective, the user has two ways or means of interacting, which are:

- Manipulation of virtual and/or real objects: This form of interaction is based on the concept known as tangible user interface [9]; under this type of interface, physical objects can be used to manipulate the virtual elements of the environment more intuitively.

- Navigation: In this medium, the apprentice moves through the real world and interacts from its location; it is the predominant type of interaction in mobile applications.

From the second perspective, the interaction is provided by a multimodal system that generates different stimuli - based on the five senses of the human being - reproduced in a virtual way to increase the immersion of the learner [10].

\section{B. AR Technology Classification}

Depending on the complexity of the system or application based on AR technology, various authors have proposed classifications. The best-known classification is the one proposed by Lens-Fitzgerald [11], considering the following levels of complexity:

- Level 0: QR codes are used that serve as hyperlinks to other content; there is no $3 \mathrm{D}$ registration or tracking of the markers.
- Level 1: Images are used as bookmarks to access the augmented item. There are two sublevels; in the first, the images are $2 \mathrm{D}$ markers that are digitized by a computer web camera; at the second level, mobile devices are used for marker recognition.

- Level 2: It is based on the geolocation of the individual through their mobile device. At this level, since the system knows where the device is located (the GPS) and in which direction the individual is looking (the compass), it is possible to augment the reality of the screen appropriately.

- Level 3. It is known as increased vision and is based on the use of display devices such as HMD (HeadMounted Display. HMD is worn on the head as part of a helmet and has a small screen in front of each of the eyes, and in the so-called HoloLens, which are a kind of lens having a sensor with which the movement of the iris is tracked.

When referring to this classification, a level 4 perhaps appears in the future, in which contact lens screens and direct interfaces to the optic nerve and brain will be used [12].

\section{MAterial AND Method}

The Systematic Literature Review (SLR) is a method that allows identifying, evaluating, and interpreting the research available in the literature relevant to a research question, subject area, or phenomenon of interest. Individual studies that contribute to an SLR are called primary studies, so a study based on a systematic review is considered a secondary study [13].

\section{A. Research Protocol}

To carry out the SLR from which the present work is derived, the formal research protocol was proposed by Kitchenham [14]. The steps are planning, execution, and dissemination of results. The purpose of the planning is to ensure that the review is systematic and rigorous, and for this, a research protocol is specified; this activity can be organized in the following activities:

- Identify the need for the review. It is about carefully summarizing the relevant information on the topic of interest and thus knowing what they have done on that topic, especially systematic reviews.

- Research questions formulation. The most important part of the systematic review is to know what we want to look for in the primary studies and answer the questions, so they must be well-posed; they are clear and consistent.

- Developing a review protocol. The rational of the SLR, research questions, sources of searches, search strategies, exclusion and inclusion criteria, and evaluation of the quality of the studies are presented.

- Evaluating a review protocol. Since the protocol is the most critical document for the performance of SLR, its validation by experts is necessary.

The second phase has the purpose of executing the activities previously defined in the protocol, which encompass the following activities:

- Identify relevant research. From the set of publications, see if they answer the research questions based on the search strategy proposed in the protocol. 
- Select primary studies. It is the process of locating the primary studies that are related to answering the research questions stated, with the help of the criteria and procedure established in the protocol

- Evaluate the quality of primary studies. From the selected studies, an evaluation criterion is applied to corroborate the quality of the studies. If necessary, those that do not follow what is established are excluded.

- Extract the relevant data. It is the process of extracting information from primary studies.

- Synthesize the extracted data. It is the process of answering the questions asked; tables and graphs can accompany this.

The final phase of the RSL is to analyze, write and disseminate the results of the review. This task can be synthesized in the following two activities.

- Write the review report. The work done using the SLR would not make sense if the information obtained and analyzed is not reported and made available to other researchers.

- Validate the review report. There are some threats to the validity of the study that it is necessary to apply some mechanisms to validate both the identified studies and their process of obtaining them.

\section{B. Planning}

In order to explore aspects related to the technological factor, a set of six research questions were established that served as a guide for our study:

- $R Q 1$. What is the number of publications related to AR research in education in the last decade?

- RQ2. What software has been used in the development of AR applications?

- RQ3. What devices have been used in AR applications?

- RQ4. What are the methodologies that have been used to develop AR applications?

- RQ5. What are the recognition methods that have been used in AR applications?

- RQ6. What are the means of interaction used in AR applications?

Once the research questions had been established, the authors proceeded to obtain the primary studies for the systematic review. The following databases were selected to search for these studies: IEEE Xplore, ACM DL, Springer and Elsevier, since these databases are related to the research area and could compile articles related to AR technology.

The search string developed uses a combination of English keywords, which are grouped into two sets; on the one hand, it is related to AR technology, in which only one keyword was identified: augmented reality; and a second set related to the educational process that is assisted with said technology: education, training, learning, teaching, evaluation, assessment, as well as a couple of these concepts that are usually used in infinitives, to teach, to learn.

For the construction of the search string, the AND operator will be used to join the two sets of keywords. In the case of the OR operator, it will be used to integrate the alternative words identified in each set; thus, the search string will be formed as: ("augmented reality") AND ("education" OR "training” $O R$ "teaching” OR "learning” OR "evaluation" $O R$ "assessment” OR “teach” OR "learn”).

The results were obtained from the digital libraries where subject to filtering by setting inclusion and exclusion criteria. These selection criteria were intended to identify those primary studies that provide direct evidence on research questions of this SLR as shown in Fig. 1.

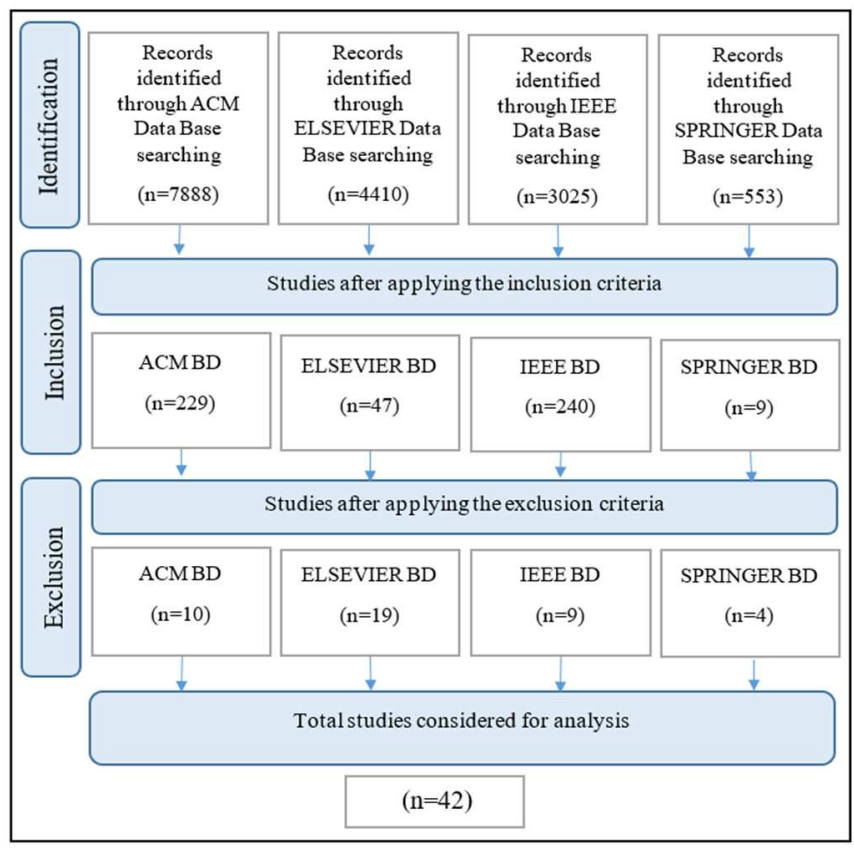

Fig. 1 Primary studies selected after applying the review protocol

The selection criteria considered for the selection of the primary studies to be analyzed listed below:

1) Inclusion criteria:

- Primary studies published in the English language.

- Primary studies published between 2010 and 2020.

- Primary studies that include at least one keyword linked to the educational process in the title.

- Primary studies that report educational initiatives in which AR is incorporated.

- Primary studies that are research articles.

2) Exclusion criteria:

- Primary articles that do not have the use of AR technology as the main element of the educational initiative.

- Articles replicated in various digital libraries; the oldest articles will be eliminated, keeping in our study the most recent.

- Articles related to the same project, articles that report partial progress will be eliminated, keeping our study the most complete.

- Articles whose content has been impossible to get.

\section{Execution}

According to the designed search string, the search process was carried out in the four selected databases; however, in the Elsevier, Springer Databases, and ACM DL the search string 
had to be slightly modified, being, in the case of Elsevier and ACM DL, as follows:

"augmented reality" AND (education OR training OR teaching OR learning OR evaluation OR assessment OR teach OR learn)

With the results obtained, the inclusion and exclusion criteria were applied. Fig. 1 illustrates the protocol defined for the selection process, with which 42 primary studies were finally obtained; these studies were analyzed to answer the research questions that are addressed in the article.

\section{RESULTS AND DISCUSSION}

This section presents the results obtained from the analysis of the 42 selected primary studies, from the four databases: 10 from ACM [15]-[24], 19 from Elsevier [25]-[43], 9 from IEEE [44]-[52], and Springer 4 [53-56]. In order to contrast our findings, three secondary studies on the use of AR in education were analyzed [57-59].

\section{A. Research Questions}

1) RQ1. What is the number of publications related to $A R$ research in education in the last decade? Based on the selected primary studies, it is observed that there has not been a constant production regarding this type of initiative in the last decade (see Fig. 2); it is possible to identify that the years with the highest frequency in publications are 2013 and 2019, with a certain tendency of growth in previous years, which coincides in the first peak [57], [58].

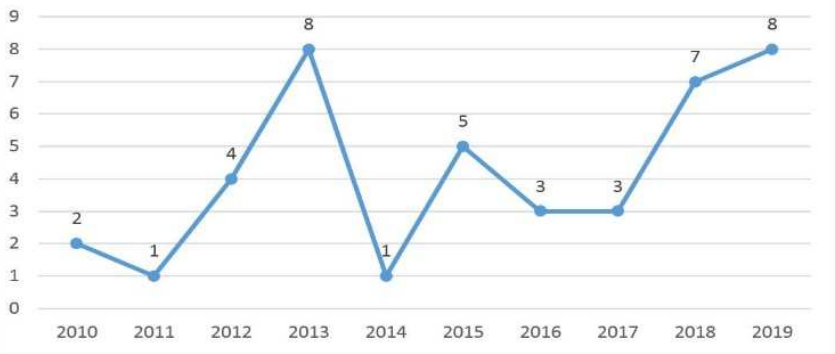

Fig. 2 Primary studies in the last decade

2) RQ2. What software has been used in the development of AR applications? Regarding the software used for the development of AR applications, it was found that most of the studies did not report the software used (36\%). Fig. 3 shows the number of studies by identified software. Based on the results obtained, it seems that a high percentage of authors do not consider the technology used to develop AR initiatives to be an important element. Note that the SRLs do not report technological aspects related to the software used in the analyzed studies [57]-[59].

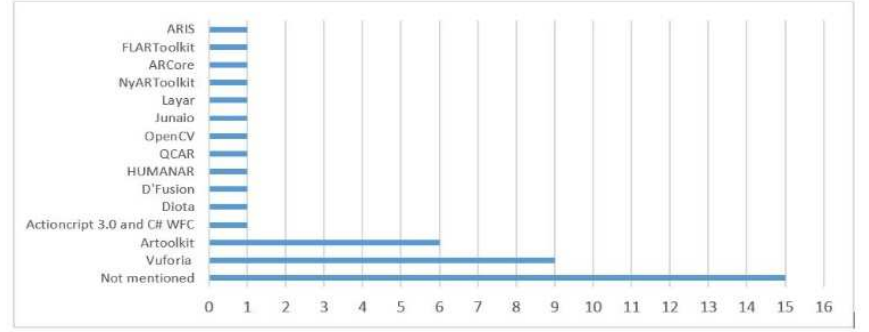

Fig. 3 Software for the development of applications based on AR technology
3) RQ3. What devices have been used in AR applications? Regarding the devices used in the primary studies, the following are mentioned: (1) mobile phone (43\%), (2) tablet $(33 \%),(3)$ webcam $(14 \%)$, (4) smart lenses or HoloLens $(10 \%)$ and (5) Helmets or head-mounted display (14\%). Our findings coincide with those reported in Si et al. [56]; the authors indicate that $60 \%$ of the analyzed studies used mobile devices. On the other hand, Akçayır and Akçayır [57] did not report a percentage of the studies that use such devices due to the popularization and advances in smartphones to make portable screens a good option for AR applications.

4) RQ4. What are the methodologies that have been used to develop AR applications? According to the selected primary studies, $92 \%$ do not report the use of any methodology for the development of AR applications; however, the remaining $8 \%$ represent three studies found at Elsevier, in which they use an educational methodology or framework for the development of their applications. Virata and Castro reported using an iterative methodology based on a Co-creation process that integrates Universal Design for Learning (UDL) as the inclusive learning approach to develop AR-based mobile applications. In Balian et al [26] the use of Extreme Programming (XP) is reported, which is a type of agile methodology under the object-oriented paradigm. For their part, Lucia et al [34] report on the use of a generic model used by instructional designers and training developers known as ADDIE - Analysis, Design, Development, Implementation and Evaluation- which represents a dynamic and flexible guide for developing. The SRLs do not report technological aspects related to the software used in the analyzed studies [57]-[59].

5) RQ5. What are the recognition methods that have been used in AR applications? With the review of the primary studies, it was found that only two of the four existing recognition methods were reported, the marker method (95\%) and the location method (5\%). Bacca et al. [59] reported that regarding the recognition method reported in the primary studies, the most widely used is that of markers (59\%), although they recognize that there is a trend in using more and more devices that allow user monitoring by using localization methods $(22 \%)$; on the other hand, the method marker-less (based on the recognition of the shapes of the objects) was reported in $12.5 \%$ of the studies.

6) RQ6. What are the means of interaction used in AR applications? To answer this question, the authors classified the means of interaction based on the five senses: visual, auditory, tactile, gustatory, and olfactory. It is worth mentioning that the interaction can be analyzed in two ways. The interactions are from the system to the user and from the user to the system. Fig. 4 illustrates the percentage of means implemented in the selected primary studies.

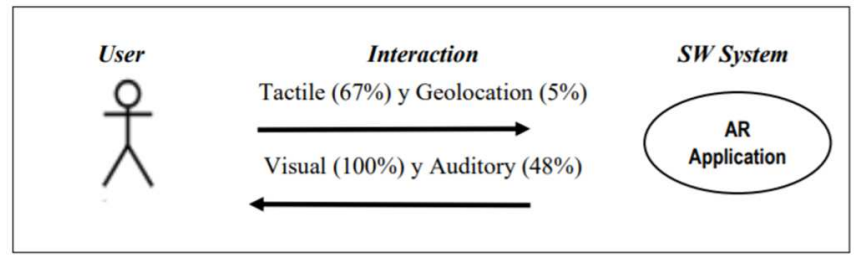

Fig. 4 Means of Interaction Identified in Primary Studies 
In da Silva [58], the authors report that regarding the interaction between the user and the system, $42 \%$ of the analyzed studies use traditional techniques (for example, buttons), $19 \%$ touch mechanisms, and one study reports the use of collaboration techniques between participants. Regarding the interaction between the system and the user, the authors report in da Silva [58] that visual representation is the predominant mechanism, and only $9 \%$ of studies report haptic mechanisms.

\section{B. Study Limitations}

Any secondary study presented in this work is subject to interpretation in all its phases and steps, so this conveys a risk of bias. To address this issue, we crosschecked the studies to each of us in order to decide whether a preliminary study is subject to discard. It allowed us to have a common consensus on the document selection process. In this sense, all borderline issues with doubt in selection and analysis were discussed to further reduce bias. Although the risk of missing relevant papers was present, the selected studies for this review represent a good enough sample of this topic.

Gray literature was not considered in the study; it was assumed that good quality grey literature on this topic would appear in journals or conference proceedings; because of this, publication bias may be present due to negative results are not usually published. Another issue to discuss is that the study did not consider documents published in a non-English language. However, this is not a limitation in our regional context; it can reflect the limitations imposed on us by the available research in this area (updated and peer-reviewed literature is published in English).

\section{IV.CONCLUSION}

AR is a technology that has become popular in the last decade; it allows combining real-world scenarios with digital objects generated by a computer system. In the educational field, AR has multiple applications in almost all areas of science. The incorporation as an instructional component has been justified by its potential as a disruptive element to motivate and promote learning.

The developed SRL has allowed us to examine and report - based on the analysis of 42 primary studies selected from 4 sources - the following: the real technological advance, in terms of the hardware artifacts used for the different interaction mechanisms, the main recognition methods had been developed, the tools or programming languages commonly used in the educational initiatives analyzed. In addition, the study findings were contrasted with findings from three previous secondary studies. It should be noted that the study carried out by the research group also considered the pedagogical aspect of the initiatives analyzed; these findings have been reported in Sosa et al. [60].

Finally, the findings of our study allow us to suppose inside the reports are not considered an important aspect to include the development methodologies of software. They are not an aspect considered in the implementation process of the software component. There are no specific methodologies that can be used and therefore reported. This hypothesis offers an opportunity for research and innovation in the knowledge area of software engineering models and methods [61].

\section{ACKNOWLEDGMENT}

We are grateful for the support provided by the Ministry of Public Education (Mexico) through the project P/PROFEXCE-2020-31MSU0098J-13. We are obliged to the National Council of Science and Technology (Mexico) through the CONACYT-CVU-1000846 scholarship.

\section{REFERENCES}

[1] J. Sánchez, Informatica Educativa, Ed. Universitaria, 1995.

[2] J. Cabero and F. García, "Realidad aumentada. Tecnología para la formación," Madrid, España, 2016.

[3] B. Furht, Handbook of augmented reality, Springer Science \& Business Media, 2011.

[4] T. Jeřábek, V. Rambousek, and R. Wildová, "Specifics of Visual Perception of the Augmented Reality in the Context of Education," Procedia - Soc. Behav. Sci., vol. 159, pp. 598 - 604, 2014, doi: 10.1016/j.sbspro.2014.12.432.

[5] R. T. Azuma, "A Survey of Augmented Reality," Presence Teleoperators Virtual Environ., vol. 4, no. August, pp. 355-385, 1997.

[6] H. Kato and M. Billinghurst, "Marker Tracking and HMD Calibration for a Video-based Augmented Reality Conferencing System," Proc. 2nd IEEE ACM Int. Work. Augment. Real., 1999.

[7] G. Kipper and J. Rampolla, Augmented reality: an emerging technologies guide to AR, 2012 .

[8] C. Portalés Ricart, "Entornos multimedia de realidad aumentada en el campo del arte," Dep. Pint., vol. Doctoral T, p. 508, 2009.

[9] H. Ishii and B. Ullmer, "Tangible bits: Towards seamless interfaces between people, bits and atoms," Conf. Hum. Factors Comput. Syst. Proc., no. March, pp. 234-241, 1997.

[10] T. Ha, Y. Chang, and N. Woo, "Usability test of immersion for augmented reality based product design," Lect. Notes Comput. Sci. (including Subser. Lect. Notes Artif. Intell. Lect. Notes Bioinformatics), vol. 4469 LNCS, pp. 152-161, 2007, doi: 10.1007/978-3-540-73011-8_17.

[11] M. Lens-Fitzgerald, (2009). Augmented Reality Hype Cycle [Online]. Available: http://www.sprxmobile.com/the-augmented-realityhypecycle.html.

[12] R. Rice, (2009). Augmented vision and the decade of ubiquity [Online]. Available:http://www.curiousraven.com/futurevision/2009/3/20/augmented-vision-and-the-decade-of-ubiquity.html

[13] M. Genero, J. A. Cruz-Lemus and M. G. Piattini, Métodos de investigación en ingeniería del software, 2014.

[14] B. Kitchenham and C. Stuart, "Guidelines for performing systematic literature reviews in software engineering," Tech. report, Ver. 2.3 EBSE Tech. Report. EBSE, 2007.

[15] C. Chen, "Integrating Augmented Reality into Blended Learning for Elementary Science Course,” Proc. 5th Int. Conf. Inf. Educ. Technol., pp. 68-72, 2017.

[16] Y. Aliev, V. Kozov, G. Ivanova, and A. Ivanov, "3D Augmented Reality Software Solution for Mechanical Engineering Education," Proc. 18th Int. Conf. Comput. Syst. Technol., pp. 318-325, 2017.

[17] A. Dünser, L. Walker, H. Horner, and D. Bentall, "Creating Interactive Physics Education Books with Augmented Reality," Proc. 24th Aust. Comput. Interact. Conf., pp. 107-114, 2012.

[18] J. Arora and N. Delhi, "Design and Evaluation of DIO Construction Toolkit for Co-making Shared Constructions," Proc. ACM Interactive, Mobile, Wearable Ubiquitous Technol., vol. 3, no. 4, pp. 1-25, 2019.

[19] Z. S. See, "Medical Learning Murmurs Simulation with Mobile Audible Augmented Reality," 2016.

[20] P. S. Medicherla, G. Chang, and P. Morreale, "Visualization for Increased Understanding and Learning Using Augmented Reality," Proc. Int. Conf. Multimed. Inf. Retr., pp. 441-443, 2010.

[21] L. Margarita and M. Herrera, "Learning Calculus with Augmented Reality and Virtual Environments,” Proc. 2019 11th Int. Conf. Educ. Technol. Comput., pp. 18-22, 2019.

[22] P. Rhienmora, K. Gajananan, and P. Haddawy, "Haptic Augmented Reality Dental Trainer with Automatic Performance Assessment," Proc. 15th Int. Conf. Intell. user interfaces, vol. d, pp. 425-426, 2010.

[23] I. Kico, "A Mobile Augmented Reality Interface for Teaching Folk Dances," 25th ACM Symp. Virtual Real. Softw. Technol., pp. 1-2, 2019

[24] R. O. Virata and J. D. L. Castro, "Augmented Reality in Science Classroom: Perceived Effects in Education, Visualization and 
Information Processing," Proc. 10th Int. Conf. E-Education, EBusiness, E-Management E-Learning, pp. 85-92, 2019.

[25] J. Bacca, S. Baldiris, R. Fabregat, and S. Graf, "Mobile Augmented Reality in Vocational Education and Training," Procedia - Procedia Comput. Sci., vol. 75, no. Vare, pp. 49-58, 2015, doi: 10.1016/j.procs.2015.12.203.

[26] S. Balian, S. K. Mcgovern, B. S. Abella, A. L. Blewer, and M. Leary, "Feasibility of an augmented reality cardiopulmonary resuscitation training system for health care providers," Heliyon, vol. 5, no. July, p. e02205, 2019, doi: 10.1016/j.heliyon.2019.e02205.

[27] A. Bin, D. Rohaya, and A. Rambli, "An Interactive Mobile Augmented Reality Magical Playbook: Learning Number With The Thirsty Crow," Procedia - Procedia Comput. Sci., vol. 25, pp. 123130, 2013, doi: 10.1016/j.procs.2013.11.015.

[28] E. Cieza and D. Lujan, "ScienceDirect Procedia ScienceDirect ScienceDirect Educational Mobile Application of Augmented Reality Based on Educational Mobile the Application of Vowel Augmented Reality Based on Markers to Improve Learning of Usage and Numbers for Children of a Kinde," Procedia Comput. Sci., vol. 130, pp. 352 358, 2018, doi: 10.1016/j.procs.2018.04.051

[29] C. Diaz, M. Hincapié, and G. Moreno, "How the Type of Content in Educative Augmented Reality Application Affects the Learning Experience," Procedia - Procedia Comput. Sci., vol. 75, no. Vare, pp. 205-212, 2015, doi: 10.1016/j.procs.2015.12.239.

[30] H. Horii and Y. Miyajima, "Augmented Reality-based Support System for Teaching Hand-drawn Mechanical Drawing," Procedia - Soc. Behav. Sci., vol. 103, pp. 174-180, 2013, doi: 10.1016/j.sbspro.2013.10.323.

[31] U. Kose, D. Koc, and S. Anil, “An Augmented Reality Based Mobile Software to Support Learning Experiences in Computer Science Courses," Procedia - Procedia Comput. Sci., vol. 25, pp. 370-374, 2013, doi: 10.1016/j.procs.2013.11.045

[32] M. H. Kurniawan and G. Witjaksono, "ScienceDirect ScienceDirect Human Anatomy Learning Systems Using Augmented Reality on Human Anatomy Learning Systems Using Augmented Reality on Mobile Application Mobile Application," Procedia Comput. Sci., vol. 135, pp. 80-88, 2018, doi: 10.1016/j.procs.2018.08.152.

[33] R. Layona, B. Yulianto, and Y. Tunardi, "ScienceDirect ScienceDirect Web based Augmented Reality for Human Body Anatomy Learning Web based Augmented Reality for Human Body Anatomy Learning," Procedia Comput. Sci., vol. 135, pp. 457-464, 2018, doi: 10.1016/j.procs.2018.08.197.

[34] M. Lucia, L. R. Okimoto, P. César, and C. Eduardo, "User Experience in Augmented Reality applied to the Welding Education," vol. 3, no. Ahfe, pp. 6223-6227, 2015, doi: 10.1016/j.promfg.2015.07.739.

[35] J. Martin-gutierrez, E. Guinters, and D. Perez-lopez, "Improving strategy of self-learning in engineering: laboratories with augmented reality," Procedia - Soc. Behav. Sci., vol. 51, pp. 832-839, 2012, doi: 10.1016/j.sbspro.2012.08.249

[36] B. Perry, "Gamifying French Language Learning: a case study examining a quest-based, augmented reality mobile learning-tool," Procedia - Soc. Behav. Sci., vol. 174, pp. 2308-2315, 2015, doi: 10.1016/j.sbspro.2015.01.892.

[37] E. Redondo, D. Fonseca, A. Sánchez, and I. Navarro, "New Strategies Using Handheld Augmented Reality and Mobile Learning-teaching Methodologies, in Architecture and Building Engineering degrees,' Procedia - Procedia Comput. Sci., vol. 25, pp. 52-61, 2013, doi: 10.1016/j.procs.2013.11.007.

[38] D. Rohaya, A. Rambli, W. Matcha, and S. Sulaiman, "Fun Learning with AR Alphabet Book for Preschool Children," Procedia - Procedia Comput. Sci., vol. 25, pp. 211-219, 2013, doi: 10.1016/j.procs.2013.11.026.

[39] P. Salinas, E. González-mendívil, E. Quintero, H. Ríos, and S. Morales, "The Development of a Didactic Prototype for the Learning of Mathematics Through Augmented Reality," Procedia - Procedia Comput. Sci., vol. 25, no. 81, pp. 62-70, 2013, doi: 10.1016/j.procs.2013.11.008.

[40] P. C. Santana-mancilla, M. A. García-ruiz, R. Acosta-diaz, and C. U. Juárez, "Service Oriented Architecture to Support Mexican Secondary Education through Mobile Augmented Reality," vol. 10, pp. 721-727, 2012, doi: 10.1016/j.procs.2012.06.092

[41] P. Stief, J. Dantan, A. Etienne, and A. Siadat, "ScienceDirect ScienceDirect Augmented Reality experiment in higher for complex system May education, appropriation in mechanical design A new methodology to analyze the functional and physical architecture of an assembly oriented product family identific," Procedia CIRP, vol. 84, pp. 197-202, 2019, doi: 10.1016/j.procir.2019.04.284.

[42] J. Yen, C. Tsai, and M. Wu, "Augmented reality in the higher education: Students' science concept learning and academic achievement in astronomy," Procedia - Soc. Behav. Sci., vol. 103, pp. 165-173, 2013, doi: 10.1016/j.sbspro.2013.10.322.

[43] M. M. Zarzuela, F. J. D. Pernas, L. B. Martínez, G. Ortega, and M. A. Rodríguez, "Mobile Serious Game using Augmented Reality for Supporting Children' s Learning about Animals," Procedia - Procedia Comput. Sci., vol. 25, pp. 375-381, 2013, doi: 10.1016/j.procs.2013.11.046.

[44] A. Ibrahim et al., "ARbis Pictus : A Study of Vocabulary Learning with Augmented Reality,” IEEE Trans. Vis. Comput. Graph., vol. 24 no. 11, pp. 2867-2874, 2018.

[45] P. Lin, Y. Huang, and C. Chen, "Exploring Imaginative Capability and Learning Motivation Difference Through Picture E-Book," IEEE Access, vol. 6, pp. 63416-63425, 2018, doi 10.1109/ACCESS.2018.2875675.

[46] A. Mart, "Supporting Teacher Orchestration in Ubiquitous Learning Environments : A Study in Primary Education," IEEE Trans. Learn. Technol., vol. 8, no. 1, pp. 83-97, 2015, doi 10.1109/TLT.2014.2370634.

[47] S. Matsutomo, T. Manabe, V. Cingoski, and S. Noguchi, "A Computer Aided Education System Based on Augmented Reality by Immersion to 3-D Magnetic Field," IEEE Trans. Magn., vol. 53, no. 6, pp. 2-5, 2017.

[48] S. Matsutomo, T. Miyauchi, S. Noguchi, and H. Yamashita, "RealTime Visualization System of Magnetic Field Utilizing Augmented," IEEE Trans. Magn., vol. 48, no. 2, pp. 531-534, 2012.

[49] M. Vazquez et al., "Enhancing Nutrition Learning Using Interactive Tools," IEEE Lat. Am. Trans., vol. 17, no. 5, pp. 751-758, 2019.

[50] D. Villar, C. Delgado-kloos, and S. Member, "Support for Augmented Reality Simulation Systems: The Effects of Scaffolding on Learning Outcomes and Behavior Patterns n," IEEE Trans. Learn. Technol., vol. 9, no. 1, pp. 46-56, 2016, doi: 10.1109/TLT.2015.2445761.

[51] M. Yang and W. Liao, "Computer-Assisted Culture Learning in an Online Augmented Reality Environment Based on Free-Hand Gesture Interaction," IEEE Trans. Learn. Technol., vol. 7, no. 2, pp. 107-117, 2014, doi: 10.1109/TLT.2014.2307297.

[52] C. T. Yeo, T. Ungi, P. U-thainual, A. Lasso, and R. C. Mcgraw, "The Effect of Augmented Reality Training on Percutaneous Needle Placement in Spinal Facet Joint Injections," vol. 58, no. 7, pp. 20312037, 2011.

[53] C. Lytridis, "Evaluation of the ARTutor augmented reality educational platform in tertiary education," Smart Learn. Environ., vol. 5, no. 1, 2018

[54] N. Nguyen, T. Muilu, A. Dirin, and A. Alamäki, "An interactive and augmented learning concept for orientation week in higher education," 2018

[55] M. E. C. Santos et al., "Augmented reality as multimedia: the case for situated vocabulary learning," Res. Pract. Technol. Enhanc. Learn., 2016, doi: 10.1186/s41039-016-0028-2.

[56] W. Si et al., "Assessing performance of augmented reality-based neurosurgical training," Vis. Comput. Ind. Biomed. Art, vol. 8, 2019.

[57] M. Akçayır and G. Akçayır, "Advantages and challenges associated with augmented reality for education: A systematic review of the literature," Educ. Res. Rev., vol. 20, pp. 1-11, 2017, doi: 10.1016/j.edurev.2016.11.002

[58] M. M. O. da Silva, J. M. X. N. Teixeira, P. S. Cavalcante, and V. Teichrieb, "Perspectives on how to evaluate augmented reality technology tools for education: a systematic review," J. Brazilian Comput. Soc., vol. 25, no. 1, 2019, doi: 10.1186/s13173-019-0084-8.

[59] J. Bacca, S. Baldiris, R. Fabregat, S. Graf, and Kinshuk, "Augmented reality trends in education: A systematic review of research and applications," Educ. Technol. Soc., vol. 17, no. 4, pp. 133-149, 2014

[60] E. Sosa, J. López y R. Aguilar, "Intervención de la Tecnología de Realidad Aumentada en la Educación: Una Revisión Sistemática de la Literatura," Revista Tecnología Educativa, vol. 7(2), 2020.

[61] P. Bourque and R. Fairley, Guide to the Software Engineering Body of Knowledge (SWEBOK V3.0). IEEE Computer Society, 2014. 